\title{
Metallurgical and Technological Request for High Purity Niobium in SRF Application
}

\author{
W. Singer \\ Deutsches Elektronen-Synchrotron (DESY), Notkestrasse 85, 22607 Hamburg, Germany; \\ waldemar.singer@desy.de
}

\begin{abstract}
Properties of niobium are analyzed regarding application in superconducting radio frequency accelerator systems. High purity niobium should meet the tough specification. Interstitially dissolved impurities like oxygen, nitrogen and carbon should be kept under few wt. ppm. Hydrogen can under definite condition significantly reduce the cavity quality factor (hydrogen decease) and should be carefully controlled during fabrication as well as during cavity treatment. The main metallic impurity is tantalum. Clusters of foreign material can essentially deteriorate the cavity performance. An eddy current scanning system with rotating table is applied for diagnostic of foreign material inclusions and cracks in niobium sheets. In addition the acceptance tests for $\mathrm{Nb}$ sheets include the RRR (RRR - Residual Resistivity Ratio) measurement, microstructure analysis, analysis of interstitial and metallic impurities, hardness measurement and tensile test.

High thermal conductivity in the cavity wall in order to guide the dissipated RF power away is required. It can be additionally improved almost by one order of magnitude during the post purification (solid state gettering) applied for Tesla Test Facility TTF cavities at DESY. The cavities are heated to $1350-1400{ }^{\circ} \mathrm{C}$ and a thin titanium layer is evaporated on the surface. Softening of $\mathrm{Nb}$ and grain growth is the main drawback of the purification annealing.
\end{abstract}

Keywords: High purity niobium, hydrogen, oxygen, nitrogen, interstitial and metallic impurities, thermal conductivity, RRR, microstructure, mechanical properties, cavity

\section{INTRODUCTION}

More and more of a new accelerating structures are based on RF superconducting cavity technology $[1,2]$. Niobium with the highest critical temperature $(\mathrm{Tc}=9,2 \mathrm{~K})$ and critical magnetic field (ca. $200 \mathrm{mT}$ ) of all pure metals is a mainly used material for the fabrication of superconducting radio frequency RF cavities. High ductility of $\mathrm{Nb}$ as bcc metal allows the fabrication of cavity parts at room temperature by deep drawing, spinning or hydroforming.

One of the main problems for RF superconducting cavities is thermal break down [2]. In order to avoid this problem, high thermal conductivity in cavity wall is needed to guide the dissipated RF power to the super fluid helium bath. The contamination of niobium can occur not only on the stage of $\mathrm{Nb}$ sheet fabrication but also on stage of cavity fabrication and treatment such as EB-welding, annealing, surface polishing and so on. Therefore, a key point of the whole procedure of cavity business is to keep a low level of contamination during all steps of fabrication and treatment.

Requirements to high purity niobium for RF cavities and its control tools are summarized below. 


\section{SPECIFICATION AND QUALITY CONTROL OF HIGH PURITY NIOBIUM FOR TTF CAVITIES}

Niobium homogeneity and purity play an important role for the RF application in order to provide the thermal stability of the cavity. Several remelting in a high vacuum electron beam furnace allow purifying the $\mathrm{Nb}$ ingot. Light impurities evaporate during this process. Four to six melting steps generally are necessary to reach the RRR $=300$ level with few wt. ppm of interstitial impurities. The melting temperature is a compromise between the maximization of purification and minimization of the material losses by evaporation. The EB melting technique in combination with advanced $\mathrm{Nb}-\mathrm{Ta}$ separation allows reaching very high RRR values (700-1000).

Intermediate and final recrystallization annealing for $1-2$ hours at $700-800^{\circ} \mathrm{C}$ in a vacuum furnace at a pressure $10^{-5}-10^{-6}$ mbar are performed in order to reach full recristallisation, uniform small grain and the mechanical properties demanded for the subsequent cavity production. The challenging task for fabrication is starting with $\mathrm{cm}$ size grain of ingot to finish with ca. $50 \mu \mathrm{m}$ uniform grain on the sheet without contamination of $\mathrm{Nb}$ on the chain of steps.

The main interstitially dissolved impurities, which reduce the RRR and the thermal conductivity, are oxygen, nitrogen, hydrogen and carbon. The influence of hydrogen on the RRR is not so significant, but the content of hydrogen should be kept small (less then 1-2 wt. ppm) to prevent the niobium hydride precipitation and degradation of the Q-value of the high RRR cavities under certain cool down conditions (hydrogen disease) $/ 2 /$.

The main substititual impurity is tantalum with a typical concentration of ca. $500 \mathrm{wt}$. ppm for high purity $\mathrm{Nb}$. Niobium and tantalum always occur in association in nature. Furthermore tantalum is difficult to separate from $\mathrm{Nb}$ because both have very similar chemical properties. A systematic investigation of the effect of Ta contents in high purity $\mathrm{Nb}$ on cavity performance was conducted in the work [3]. No systematic dependence of quench field level on Ta contents was seen for 160 wt. ppm. $<\mathrm{Ta}<1300$ wt. ppm at least for accelerating gradients $<30 \mathrm{MV} / \mathrm{m}$.

The niobium specification used for cavity of TESLA technology [1] is listed in the table 1 .

The acceptance tests of $\mathrm{Nb}$ sheets include the RRR measurement, microstructure analysis, analysis of interstitial and metallic impurities, hardness measurement, tensile test, examination of the surface roughness and search for clusters.

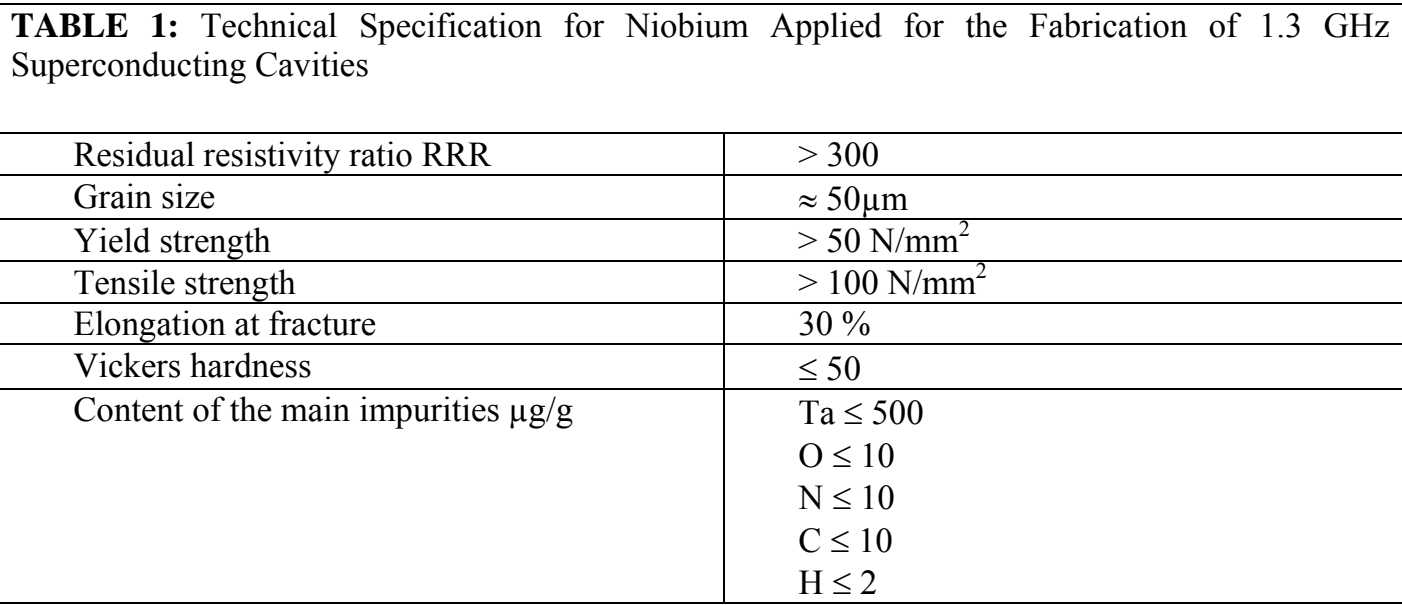


A frequent limitation of the accelerating gradient Eacc in the cavities is due the thermal instabilities caused by defects (cluster of foreign materials, micro cracks, rests of the oxides and so on). Temperature mapping reveals isolated hot spots away from the EB welding seam and demonstrates the importance of the search for defects in $\mathrm{Nb}$ sheets.

Some methods of nondestructive diagnostic as X-ray radiography, neutron radiography, neutron activation analysis NAA, X-ray fluorescence analysis, ultrasonic- and eddy current inspection has been taken into consideration. The eddy current method was chosen as most suitable for $100 \%$ sheet scanning (rather fast, sensitive to different sorts of defects with a high resolution). An eddy current scanning system was developed [1]. The system rotates the $\mathrm{Nb}$ sheet continuously; the scanning probe is placed like the tangential arm of a record player. The applied two frequency principle gives the possibility to separate the surface and bulk signal contribution. All $\mathrm{Nb}$ sheets foreseen for TESLA Test Facility cavities (TTF) were eddy current tested (about 1000). Sheets with irregularities (for example see Fig.1a) were sorted out. Open communication with $\mathrm{Nb}$ manufacturer led to continues reduction of the number of detected defects from series to series of $\mathrm{Nb}$ production.

A supplemental nondestructive identification of defects could be done by X-ray Fluorescence Analysis SURFA and neutron activation analysis NAA. The first method giving a penetration depth between few $\mu \mathrm{m}$ and few hundred of $\mu \mathrm{m}$ is more efficient for analysis of layers close to the surface (Fig.1b ). NAA delivers the information about bulk $\mathrm{Nb}$ and demonstrates very high sensitivity to Ta inclusions in $\mathrm{Nb}$.

Further improvement of the scanning system for detection of smaller defects in niobium can be done by the SQUID based methods. SQUID sensors are more sensitive in comparison to conventional eddy current pick up coils $/ 4 /$.

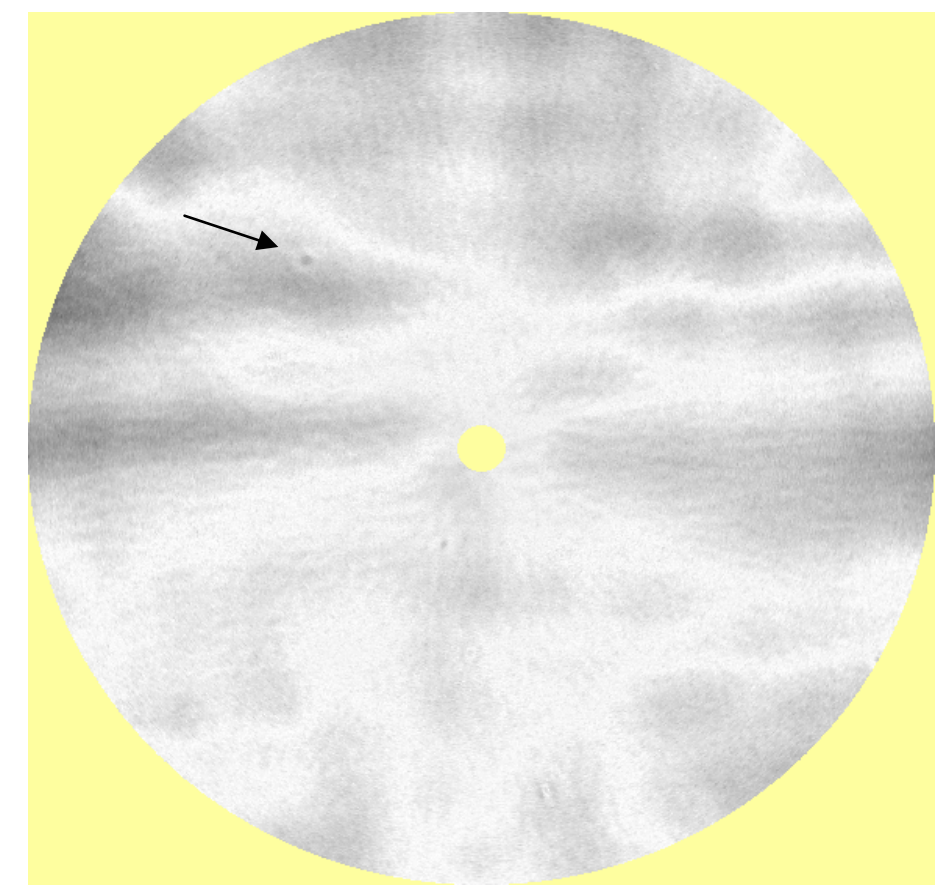

FIGURE 1a. Eddy current scanning test of the Nb-sheet. Arrow indicates the suspicious spot. 


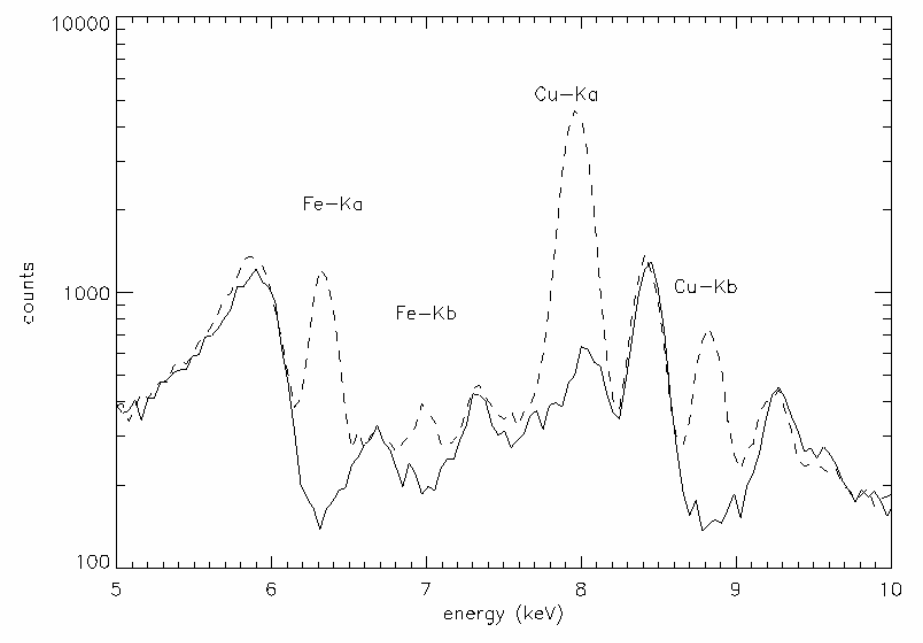

FIGURE 1b. SURFA (Synchrotron Radiation Fluorescence Analysis). Spectrum of K-lines at the spot area (dashed line) in comparison with spot free area (full line).

\section{THERMAL CONDUCTIVITY AND RRR OF HIGH PURITY NIOBIUM FOR RF CAVITY}

Interstitially dissolved gases and lattice defects act as scattering centres for unpaired electrons and reduce the thermal conductivity and RRR. A cryogenic thermal conductivity measurement, a DC RRR measurement and a non-destructive AC RRR measurement are very useful for diagnostic of material quality level [5].

For measuring the thermal conductivity at cryogenic temperatures the steady state method is mostly applied. The conductivity $\mathrm{k}$ is given by the formula

$$
Q=-k \bullet A \bullet \frac{d T}{d x}
$$

By measuring the temperature difference $\mathrm{dT}$ over a distance $\mathrm{dx}$, the heat input $\mathrm{Q}$ and the cross section A of the sample, the thermal conductivity $\mathrm{k}$ can be derived.

Some measurement results for niobium with RRR between 100 and 800 get on DESY set up are shown in Fig. 2. The strong dependence of thermal conductivity on $\mathrm{Nb}$ purity (RRR) without the indication of phonon peak can be seen.

In practice, the simplified relationship between RRR and thermal conductivity $\lambda(4.2 K) \approx R R R / 4$ allows the employment of RRR control for rough estimation of the thermal conductivity. The empirical formula (2) can be used to estimate more precise the thermal conductivity of superconducting niobium in a wide temperature range, if the RRR and grain size $\mathrm{G}$ is known [6]:

$$
\lambda(T, R R R, G)=R(y) \cdot\left[\frac{\rho_{295 K}}{L \cdot R R R \cdot T}+a \cdot T^{2}\right]^{-1}+\left[\frac{1}{D \cdot \exp (y) \cdot T^{2}}+\frac{1}{B \cdot G \cdot T^{3}}\right]^{-1} \ldots
$$

First term in (2) describe the scattering of electrons by impurities, lattice defects and phonons, the second term describe the scattering of phonons by the electrons and grain boundaries.

RRR strongly depends on the sort and concentration of impurities in $\mathrm{Nb}$. There are several empirical formulas, which describe the influence of different impurity elements on RRR. For example, RRR dependence on oxygen, nitrogen and carbon contents can be calculated as reported in [7] and the results are shown in Fig. 3 (it is assumed that carbon content $=2 \mathrm{wt}$. ppm). It can be easily seen that for RRR $\approx 300$ the content of oxygen and nitrogen is close to $6-8 \mathrm{wt}$. ppm. 


$$
\begin{gathered}
R R R=\frac{\rho(300 K)}{\rho(10 K)+\sum_{i=1}^{4} \frac{\partial \rho_{i}}{\partial C_{i}} C_{i}} \cdots \text { (3) } \\
\rho(10 K)=8.7 \times 10^{-9} \Omega \bullet \mathrm{cm} \rho(300 K)=1.46 \times 10^{-5} \Omega \bullet \mathrm{cm} .
\end{gathered}
$$

For destructive RRR control of niobium a 4-point DC RRR measurement system is mostly used. RRR is defined as

$$
R R R=\frac{\rho(295 K)}{\rho(4.2 K)}
$$

Niobium has high critical temperature $\mathrm{Tc}=9.3 \mathrm{~K}$, what makes difficulties to measure the specific resistivity at $4.2 \mathrm{~K}$ directly. Two procedures have been developed. One way is to suppress the superconductivity by exposing $\mathrm{Nb}$ to an external magnetic field of $\mathrm{H}>\mathrm{Hc}$ (a homogeneous magnetic field up to 1 Tesla parallel to sample is necessary). The second procedure: - measuring the electrical resistivity above Tc but close to it and extrapolation to $4.2 \mathrm{~K}$ for example using the formula:

$$
R R R=\frac{U(295 K)}{U(9.3 K)-U(295 K) \cdot 2.4587 \bullet 10^{-4}} \quad \cdots
$$

For non-destructive RRR control in particular after cavity post purification an AC method was developed (Fig. 4). The technique involves two concentric coils positioned close to the object. A current with definite frequency is created in the primary coil; the magnetic field of this coil induces eddy current in the metal. The signal appeared in the pick up coil is a function of the material impedance. The superconductivity jump of the signal is utilized for RRR identification. To eliminate the inductive voltage, which the primary coil creates in the pick up coil without test material, two identical contrary directed pick up coils are used. Accuracy of about 10\% can be achieved by using of standard samples for calibration. With the eddy current RRR test system, the routinely

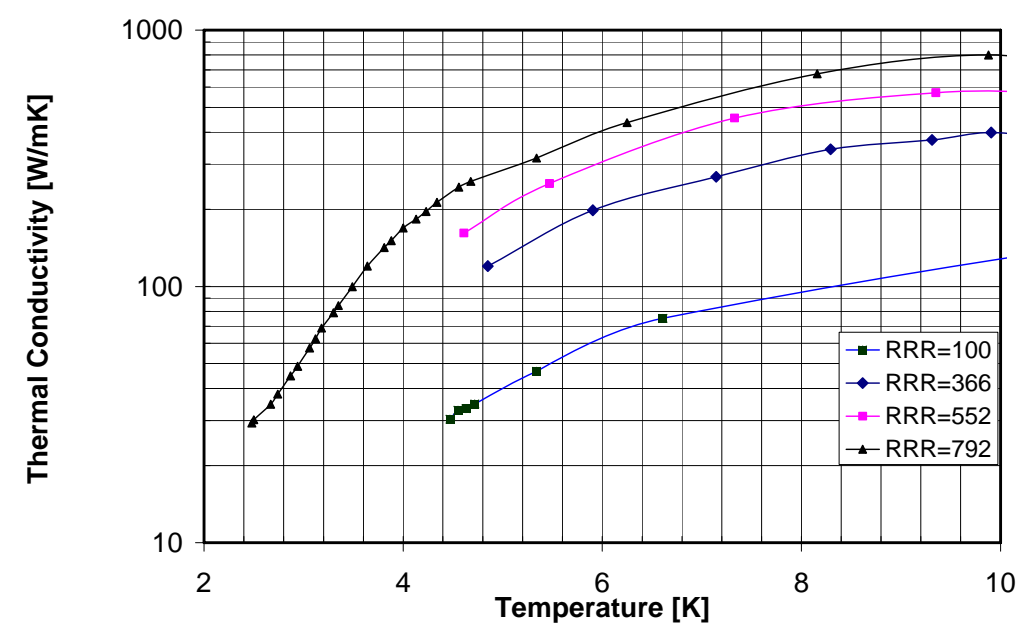

FIGURE 2. Niobium thermal conductivity vs RRR 


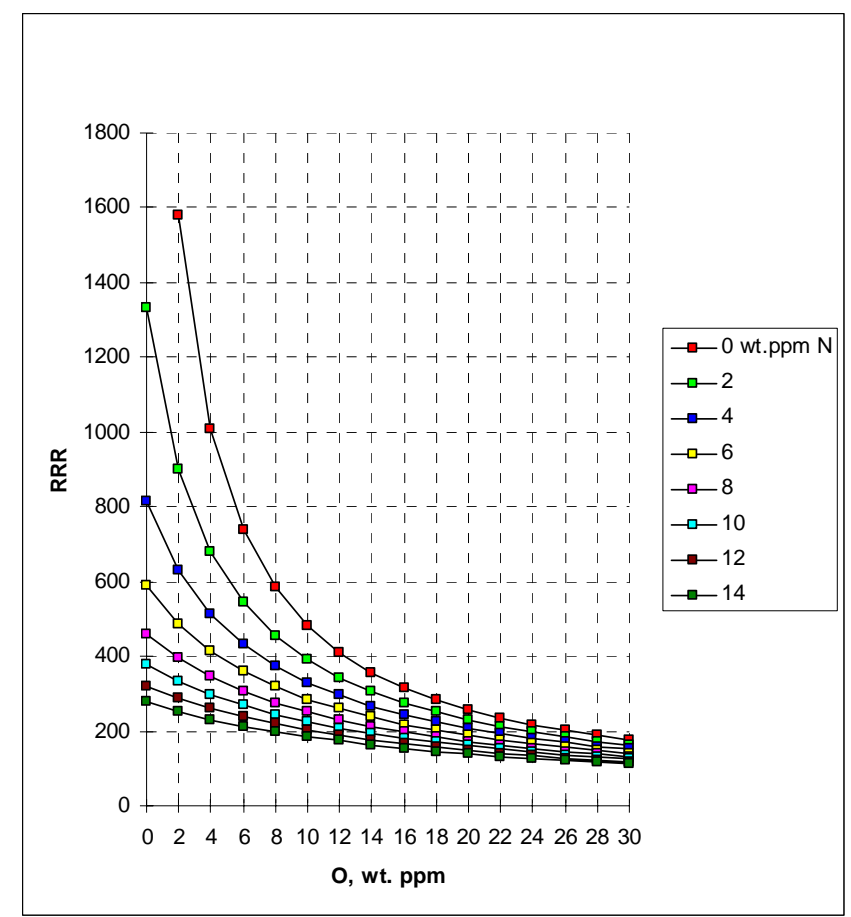

FIGURE 3. Calculated RRR versus oxygen and nitrogen content.

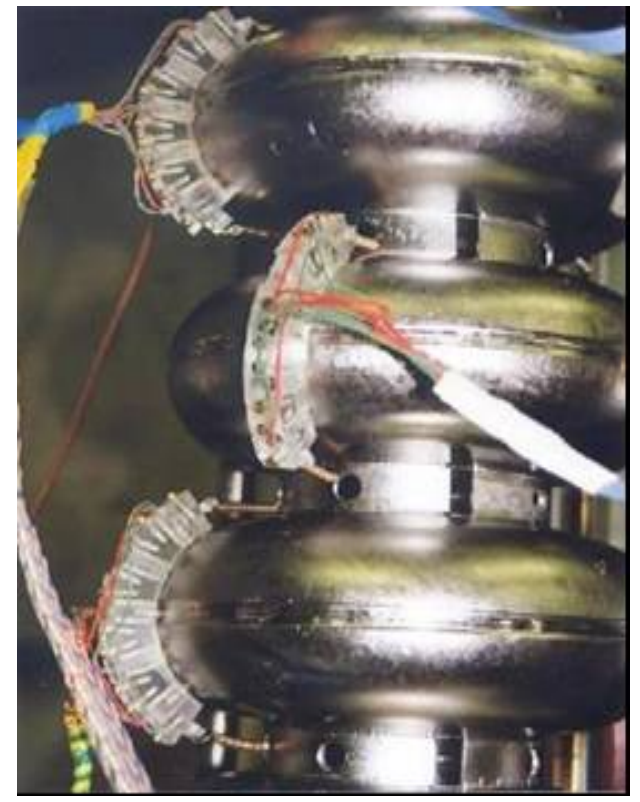

FIGURE 4. Non-destructive RRR test system

RRR control of the TTF cavity during its cool down or heat up for RF test in the vertical cryostat is done. The collected data of RRR versus accelerating gradient Eacc for TTF cavities are shown in Fig. 5. For cavities treated by buffered chemical polishing (BCP) the tendency to square root dependence of the accelerating gradient on the RRR can be seen. It seems that the Eacc for EP treated (electopolished) cavities is independent on RRR within RRR values of 200-600. 


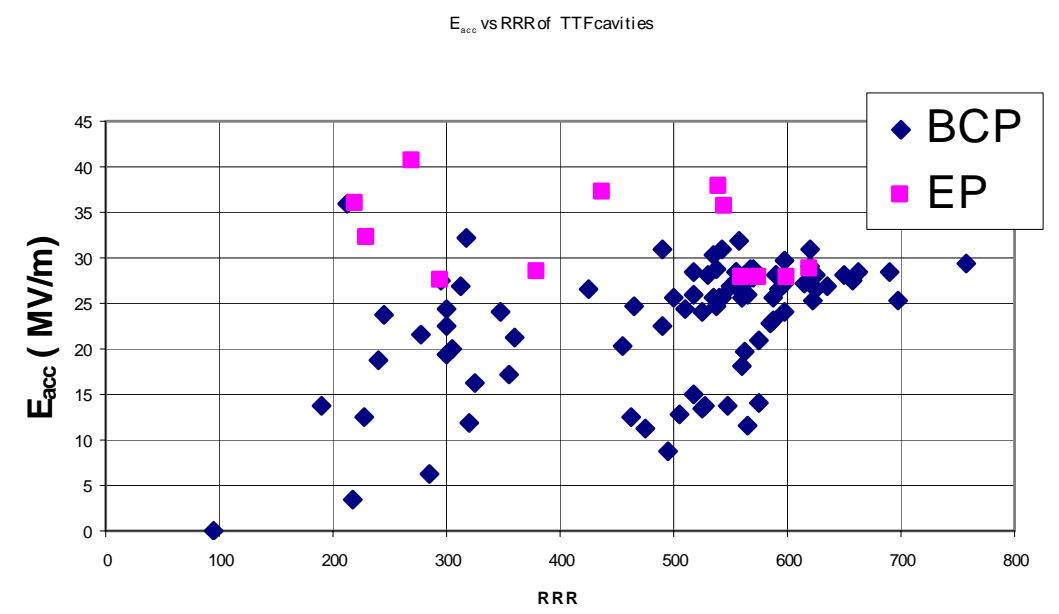

FIGURE 5. TTF 9-cell cavity performance vs RRR

\section{CONTAMINATIONS DURING CAVITY FABRICATION}

Adequate procedures that allow reaching in the melted ingot the few wt. ppm level of interstitial contamination have been developed by some $\mathrm{Nb}$ producers. This level should be kept during the whole procedure of cavity fabrication and treatment. The contaminations can occur in some cases like machining, EB welding, surface polishing etc. Some examples are mentioned below.

\section{Contamination during machining}

Examples concerning the contamination due to machining can be seen in Table 2 . High pressure water jet cutting contaminated the sample's surface by oxygen, electro erosion (EDM) contaminated the surface by oxygen and the bulk by hydrogen. Milling is probably the most appropriate procedure accompanied by small contamination.

\begin{tabular}{|l|r|r|r|r|r|r|r|r|r|r|r|r|r|}
\hline \multicolumn{9}{|c|}{ TABLE 2. Contamination during machining } \\
\hline & \multicolumn{9}{|l|}{ Water cutting } & \multicolumn{1}{l|}{ Electroerosion } & \multicolumn{3}{l|}{ Milling: } \\
\hline Sample & RRR & O, ppm & N, ppm & H, ppm & RRR & O, ppm & N, ppm & H, ppm & RRR & o, ppm & N, ppm & H, ppm \\
\hline Nb sheet, & 275 & 67 & 7 & 1 & 286 & 522 & 13 & 9 & 265 & 32 & 8 & 1 \\
\hline degreased & & 96 & 11 & & & 535 & 13 & & & 19 & 8 & \\
\hline Nb sheet, & & 9 & 3 & 1 & & 6 & 7 & 6 & & 8 & 9 & 1 \\
\hline $20 \mu$ BCP & & 12 & 7 & & & 8 & 3 & 7 & & 11 & 6 & \\
\hline
\end{tabular}

\section{Contamination during E-beam welding}

The RRR degradation of high purity $\mathrm{Nb}$ in the $\mathrm{EB}$ welding chamber with ultra high vacuum (total pressure $<10^{-5} \mathrm{mbar}$ ) is investigated in [8]. This opportunity becomes realistic after the installation of new EB welding equipment at DESY, which can be operated in the pressure range of $10^{-5}-10^{-8}$ mbar. If we take into consideration only the welding seam itself, we can see that the RRR degradation started from the pressure higher than ca. $5 \times 10^{-6} \mathrm{mbar}$, as shown in Fig. 6 . At the pressure below $5 \times 10^{-6} \mathrm{mbar}$, the RRR in the welding seam can be even improved from ca. 350 up to 370-380. The 
improvement of RRR reaches the highest value for the pressure region $10^{-8}-5 \times 10^{-7}$ mbar. The RRR improvement almost does not depend on the pressure in this region.

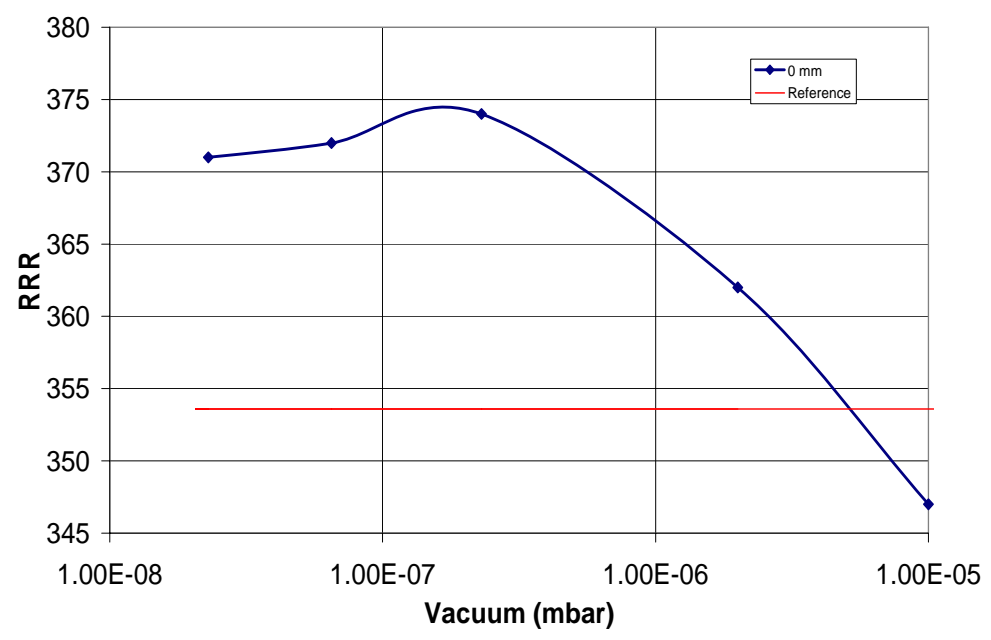

FIGURE 6. RRR in the welding seam versus pressure in the welding chamber of DESY EB facility

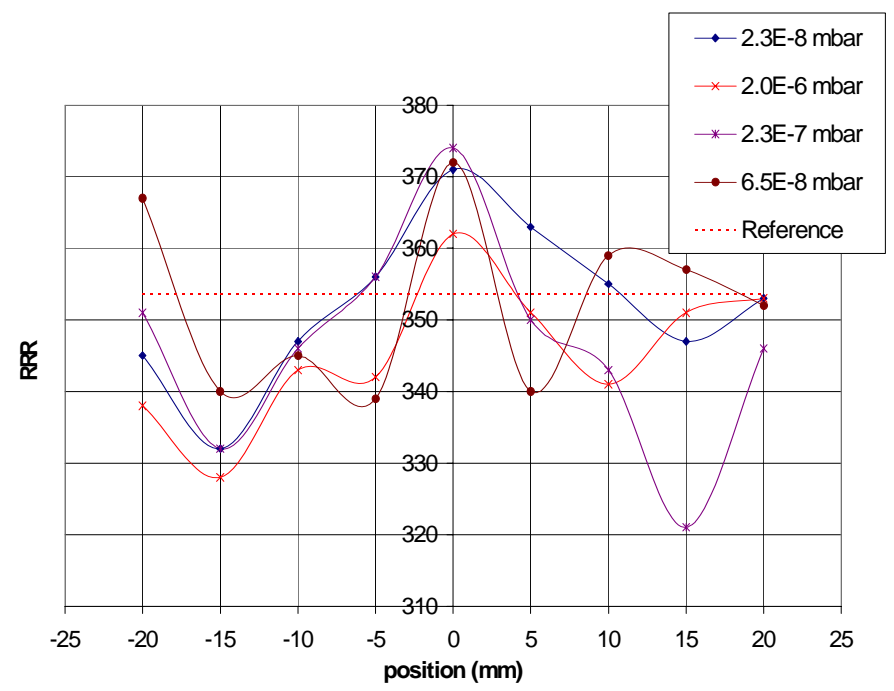

FIGURE 7. RRR in the EB welding area versus distance from the welding seam at different pressures of DESY EB facility

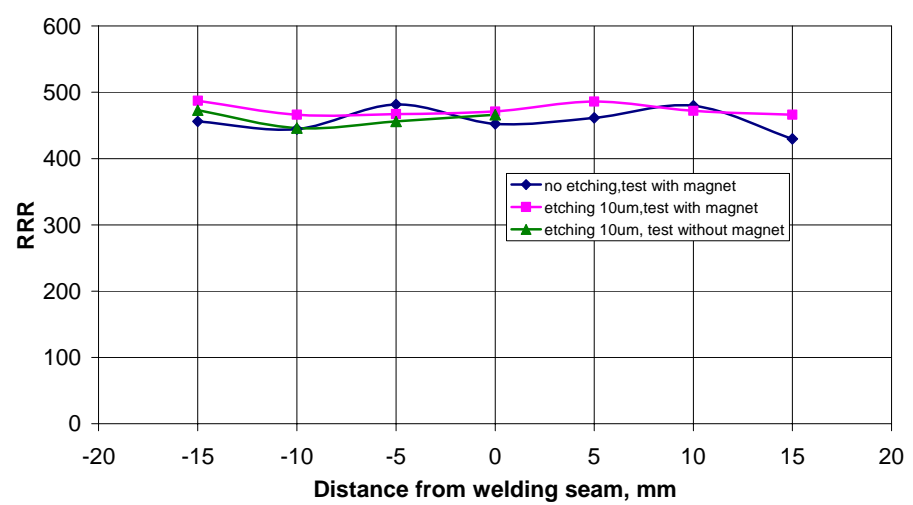


FIGURE 8. RRR distribution in Nb sample EB welded at pressure 5x10-5 mbar after vaporizing of high purity niobium

This means that the effort to reach the vacuum better than $5 \times 10^{-7} \mathrm{mbar}$ is not necessary. On the other hand it can be concluded that the RRR degradation takes place in the heat-affected area, as shown in Fig. 7. It can be seen that RRR degradation reaches the peak value in the region about $10-15 \mathrm{~mm}$ away from the welding seam (from 350 to 310-330). Good correlation between RRR degradation in the welding region and contents of interstitial impurities in the same area is observed [9].

It is worthwhile to point out that the RRR degradation in the EB welding area can be very good suppressed even for the common vacuum conditions of ca. $5 \times 10^{-5}$ mbar by vaporising of high purity $\mathrm{Nb}$ in the welding chamber before welding. (see Fig. 8).

\section{Contamination during centrifugal barrel polishing CBP (tumbling)}

A new option of centrifugal barrel polishing technique $\mathrm{CBP}$ for $\mathrm{Nb}$ superconducting cavities was developed in last few years at DESY [10]. Unfortunately, this procedure is accompanied by hydrogen contamination of niobium. Fig. 9 shows the hydrogen contamination in single cell cavity from iris to iris before and after degassing annealing. The removal rate of $\mathrm{Nb}$ layer during barrel polishing has the maximum at equator and the minimum at iris. This aspect is reflected in similar behavior of hydrogen content distribution from equator to iris. The CBP takes place in mix of water and grinding stones. $\mathrm{CBP}$ as a sort of grinding remove the protecting niobium pent oxide surface layer and make the way free for hydrogen contamination from water. Using of hydrogen free liquid can be very helpful regarding of this problem

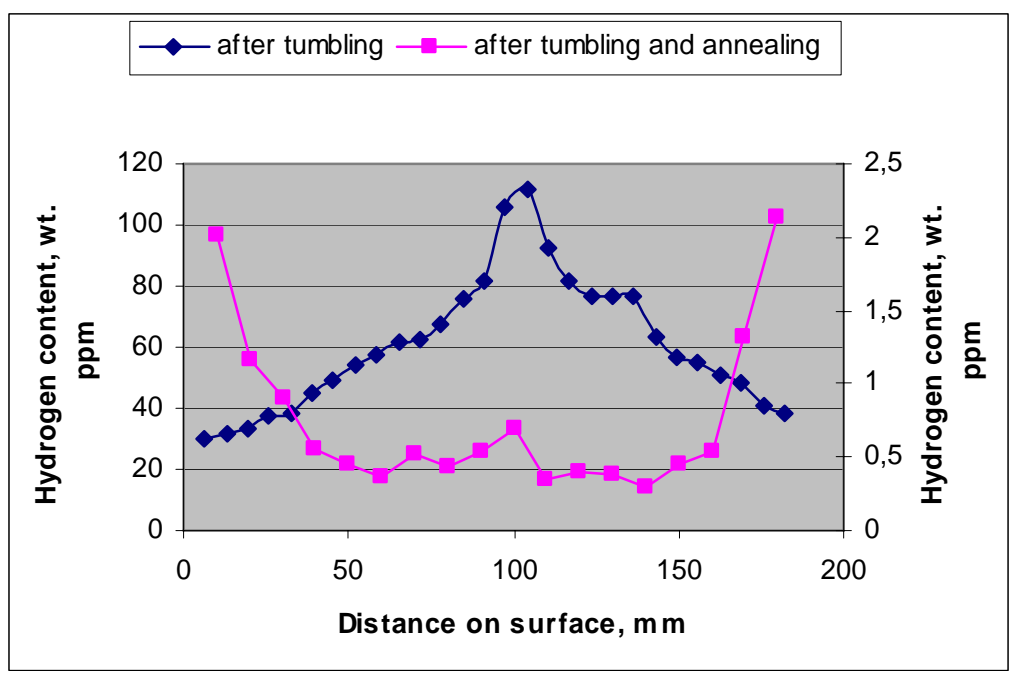

FIGURE 9: Hydrogen distribution in the single cell cavity after CBP (tumbling) and annealing

\section{HOW GET RID THE CONTAMINATION?}

As it was shown above it is very difficult to avoid interstitial contamination in some cases. Can be such contaminations cured? Efficiency of additional purification of niobium depends on the sort of contamination.

\section{Hydrogen Contamination}


The hydrogen degassing can be reached by common annealing. The hydrogen partial pressure versus temperature shows normally two peaks around $350^{\circ} \mathrm{C}$ and $600-650^{\circ} \mathrm{C}$. It can be assumed that the peak at $350^{\circ} \mathrm{C}$ is caused by surface trapped hydrogen, while the other peak indicates the bulk hydrogen contaminations. There are some differences in the opinions concerning of the optimal hydrogen degassing temperatures. Annealing at $600^{\circ} \mathrm{C}$ for $10 \mathrm{~h}$ was proposed in the work [12]. The investigation [13] shows that the heat treatment of $800^{\circ} \mathrm{C}$ for five hours will result in the largest depletion of hydrogen from the material as $600^{\circ} \mathrm{C}$ for $10 \mathrm{~h}$. The annealing at $800^{\circ} \mathrm{C}$ for two hours is applied for TTF cavities which of our opinion is rather optimal and allows to reach the hydrogen level of $<1 \mathrm{wt}$. ppm. Annealing at $800{ }^{\circ} \mathrm{C}$ has some advantages: the degassing of the oven is rather fast, and the niobium becomes completely recrystallized at this temperature. In addition, the lattice presents fewer defects after such annealing. The improved lattice is not so sensitive to further hydrogen contamination.

\section{Oxygen, Nitrogen and Carbon Contamination}

One needs very high temperature and very low partial pressures in order to degas the oxygen and nitrogen [14]. Such annealing makes not much sense for cavity application. Purification heat treatment (solid state gettering) is more reasonable. In this case niobium is heated together with getter material in order to vapor deposit the getter atoms on the surface of niobium. Important is that the bonding enthalpy of the getter material (like Ti, Y, Zr, Hf ) with oxygen, nitrogen or carbon is higher as of $\mathrm{Nb}$. Pure titanium is applied for TTF cavities. The cavities were heated at $1350-1400{ }^{\circ} \mathrm{C}$ for 4 hours. During refining the interstitial impurities oxygen, nitrogen, carbon moves to the surface and build compounds with Ti. The purity of niobium can be doubly improved (from RRR300 to RRR600), while common annealing without Ti reduces it.

A definite distribution of the interstitial impurities in the cross section of the cavity wall takes place after such purification. The concentration of $\mathrm{O}, \mathrm{N}, \mathrm{C}$ decreases from the center to the surface (calculated in [7]). Similar behavior of RRR is to expect. For estimating the RRR distribution of purified sample, a rough method was proposed in the work [15]. A niobium sample after purification heat treatment was etched layer by layer, and the RRR was measured after each step. The RRR distribution of such sample is shown in Fig.10. It can be clearly seen, that the RRR close to the surface is much higher than that inside the sample, which is in good correlation with the distribution of interstitial impurities in the cross section of niobium after post purification.

Post purification increases RRR and allows reaching higher gradients after BCP treatment (Fig. 5). Nevertheless it has two drawbacks that narrowed the application of this procedure: firstly it reduces the cavity stiffness caused by grain growth (reduces the breaking elongation, yield and tensile strength), what can be critical for keeping undisturbed the resonant frequency of cavity cells. Secondly titanium diffuses into niobium during post purification. The diffusion depth of Ti is about 60-80 $\mu \mathrm{m}$ (Fig.11). As it can be expected this penetration depth is deeper in grain boundaries than in grains [16] (see Fig.12). This polluted layer of Ti compounds must be removed (as an rule chemically). 


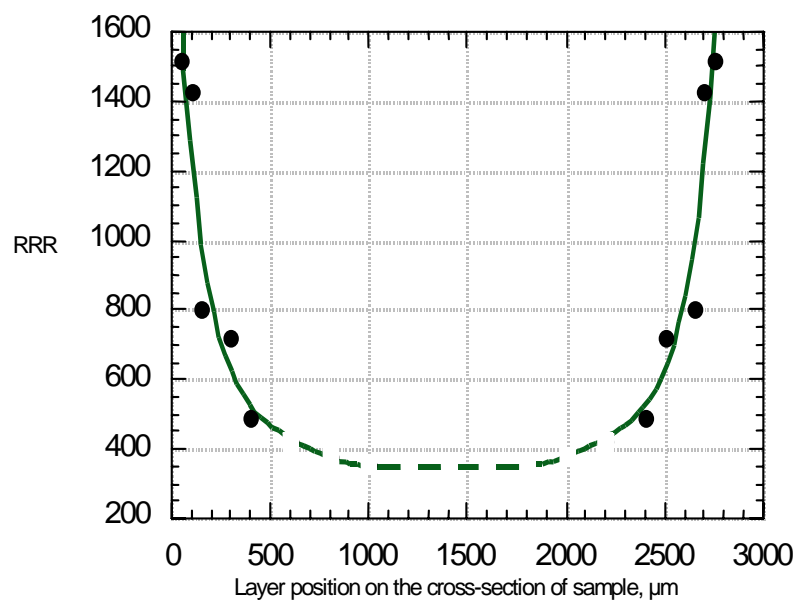

FIGURE 10. RRR distribution inside of $\mathrm{Nb}$ sheet after post-purification

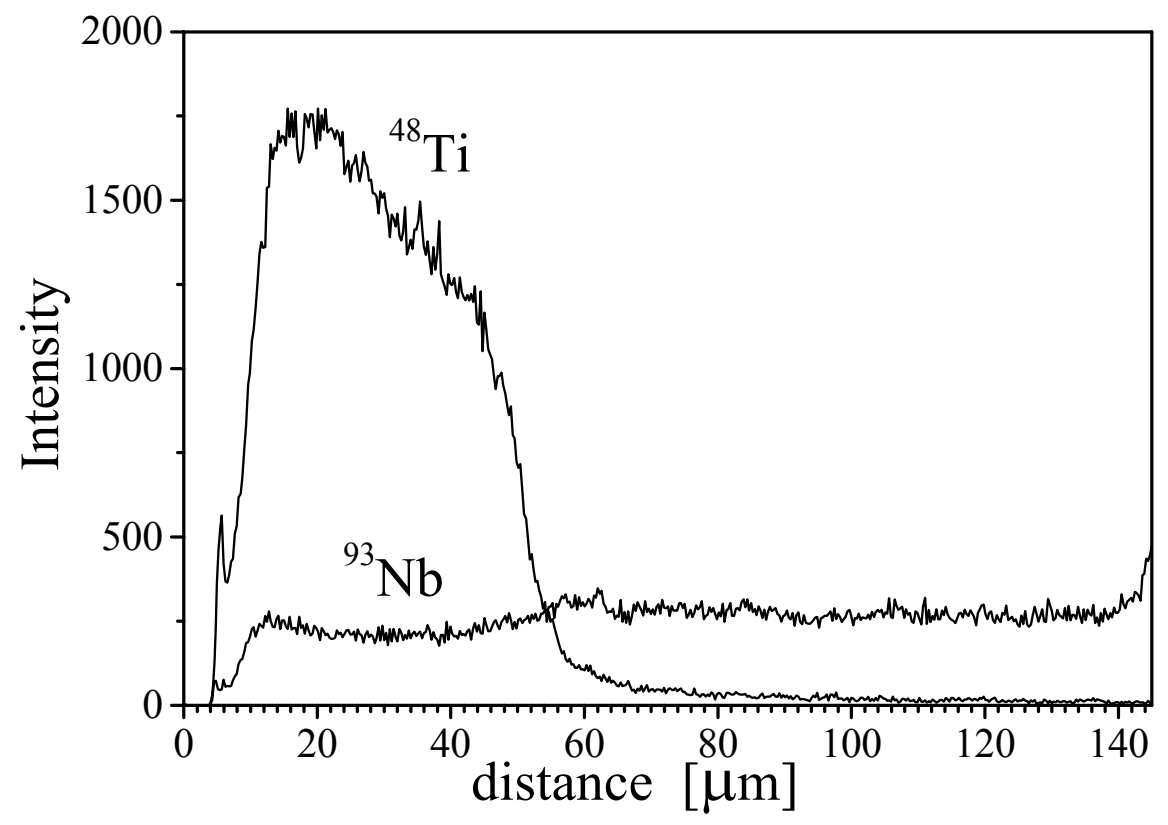

FIGURE11. SIMS linescanes measured on the cross-section of the niobium covered by Ti layer

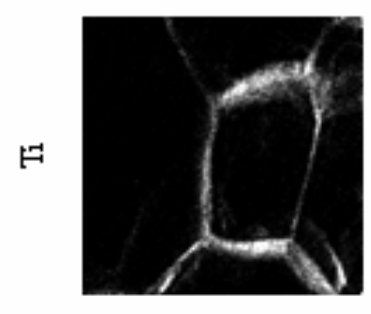

籍量

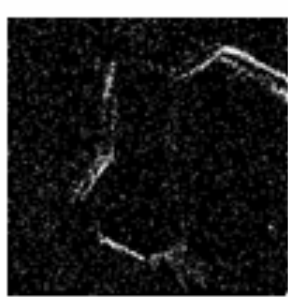

으

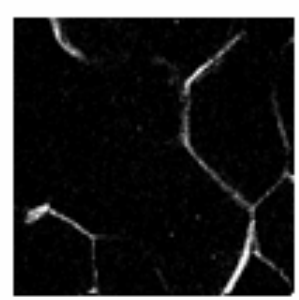

2

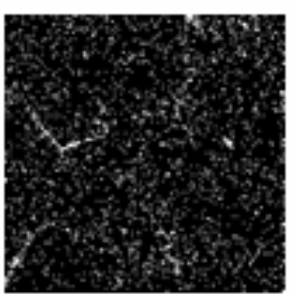

马

FIGURE12. Composition maps of the samples post purified with Ti and chemically etched for different time. Diffusion of Ti in the grain boundaries can be seen. 


\section{LARGE GRAIN NIOBIUM}

The power of the niobium melting equipment is growing at the industry steadily. Ingots with diameters of $300-500 \mathrm{~mm}$ can be produced today. Such big diameter allows in principle simply cut discs of appropriate thickness from the ingot und produce from it cavities by common deep drawing and electron beam welding. This fabrication way was proposed by colleagues of JLab [17, 18]. It seams that large grains of the ingot does not make problems. The cavities are leak tight and steps appeared on grain boundaries after deep drawing can be removed by grinding. First experiences have shown that large grain niobium cavities can demonstrate high accelerating gradients up to $35 \mathrm{MV} / \mathrm{m}$. The grain can be additionally enlarged by optimization of EB melting parameters. The aim in the longer term is creation of the single crystals with dimensions sufficient for cavity fabrication. For example a TESLA shape cavity needs discs of $265 \mathrm{~mm}$ diameter. Fabrication of such large single crystals is a challenging but not unrealistic task for the industry. A single crystal option could lead to a real break through in the cavity technology. On one hand it seems to be a cost effective solution because the long chain of fabrication steps from ingot to $\mathrm{Nb}$ sheet (containing danger of additional material contamination) will disappear. On the other hand it could allow reaching very high accelerating gradient by simplified quality control and treatment procedure. For example the eddy current scanning and electropolishing, could be probably avoided. Very high potential of the single crystal option is already demonstrated on a small cavity of the frequency ca. 2.2 GHz fabricated from two single crystal discs. Accelerating gradient of ca. $46 \mathrm{MV} / \mathrm{m}$ was achieved [18]. This very interesting development will definitely bring in next years new exciting aspects to the cavity business.

\section{SUMMARY}

1. Low impurity contents of the raw material, stringent conditions during electronbeam melting of the ingots, forming, sheet rolling, machining and recrystallization heating are required to niobium for cavities. Adequate procedures have been developed by some $\mathrm{Nb}$ producers that allow reaching in the sheet material the few wt. ppm level of interstitial impurity.

2. Clean and well controlled conditions during deep drawing, electron-beam welding, machining, annealing, grinding etc. allow in most cases keeping the content of interstitial impurities in niobium on an acceptable low level for high performance of RF cavities.

3. Additional cavity purification can reduce the content of interstitial impurities of niobium. Unfortunately cavity solid state gettering with $\mathrm{Ti}$ causes two disadvantages: reducing of cavity stiffness and Ti diffusion into niobium.

4. Cavity fabrication from large grain discs cut directly from EB melted niobium ingot is a new and promising development. Melting of the niobium ingot with sufficiently large single crystals is a challenge for industry, but it could lead to a real break through in the cavity technology.

\section{REFERENCES}

[1] B.Aune, ..., Superconducting TESLA cavities, Physical Review, Special Topics-Accelerators and Beams, Volume 3, 092001(2000). 
[2] H. Padamsee, J. Knobloch, T. Hays. RF Superconductivity for Accelerators, John Willey \& Sons, Inc., New York, 1998, P.199

[3] P. Kneisel, G. Ciovati, G. R. Myneni, W.Singer, X. Singer, D. Proch, T. Carneiro. Influence of Ta content in high purity niobium on cavity performance. Particle Accelerator Conference, USA, Mai 16-20, 2005

[4] F. Schölz, A. Farr, E.Wappler, M. Mück, A. Brinkmann, W. Singer. SQUID-based scanning system for detecting defects in Nb sheets for RF cavities. Proceedings of $12^{\text {th }}$ Workshop on RF Superconductivity, SRF- 2005. 10-15 July 2005, Ithaca, USA.

[5] H.M.Wen, W.Singer, D.Proch, L.Z.Lin, L.Y. Xiao, "Cavity RRR Test with Eddy Current Method for TESLA Test Facility", Proceedings of the 18th International Cryogenic Engineering conference(ICEC18), Mumbai, India 2000

[6] F. Koechlin, B. Bonin, Parametrisation of the Niobium Thermal Conductivity in the Superconducting State, Supercond. Sci. Technol.9(1996)453-460, IOP Publishing Ltd.

[7] W. Singer, Some Aspects of Diffusion in Niobium by High Temperature Gettering, TESLA Report, 1996, URL http://tesla.desy.de/new_pages/TESLA/ TTFnot96.html

[8] W.Singer, X.Singer, J.Tiessen, H.M.Wen, F.Schölz, RRR Degradation and Gas Absorption in the Electron Beam Area of High Purity Niobium", International Workshop on Hydrogen in Materials \& Vacuum Systems, AIP Proceedings 671, American Institute of Physics, Melville, New York 2003

[9] S. Anakhov, X. Singer, W. Singer, H. Wen. Gas Distribution in High Purity Niobium EB Welded in UH Vacuum. International Symposium on Hydrogen in Matter (ISOHIM 2005) Uppsala, Sweden, 13-17 June 2005.

[10] D. Proch. G. Issarovitch. X. Singer. D. Reschke. W. Singer. Development of Centrifugal Barrel Polishing and Single - Point Burnishing Methods for Treatment of Cavities RF-Surface. Proceedings of $11^{\text {th }}$ Workshop on RF Superconductivity, SRF- 2003. 8-12 September 2003, Lübeck, Germany.

[11] T. Higuchi, K .Saito. DEVELOPMENT OF HYDROGEN-FREE EP AND HYDROGEN ABSORPTION PHENOMENA. Proceedings of 11th Workshop on RF Superconductivity, SRF- 2003. 8-12 September 2003, Lübeck, Germany.

[12] G.R. Myneni, S.R. Agnew. Elasto-Plastic Behaviour of high RRR Niobium: Effects of Crystallographic Texture, Microstructure and Hydrogen Concentration. International Workshop on Hydrogen in Materials \& Vacuum Systems, AIP Proceedings 671, American Institute of Physics, Melville, New York, 2003

[13] C. Chapman, Niobium Heat Treatment Study Report. Fermi National Accelerator Laboratory Report, TD-04036, 8-13-2004.

[14] K. Schutze, O. Bach, D. Lupton and F. Schreiber. Purification of Niobium. Niobium, Proceedings of the International Symposium. November 8 - 11, 1981. San Francisco, USA.

[15] H.M. Wen, W. Singer, D.Proch, TESLA Report 98-02(1998), DESY, Hamburg, Germany, URL http://tesla.desy.de/ new pages / TESLA/TTFnot98.html

[16] A. Bernasik, K. Kowalski, X. Singer, W. Singer, Bulk and Grain Boundary Diffusion of Titanium in High Purity Niobium, TESLA Report 2001 - 34, DESY, Hamburg, Germany, URL http://tesla.desy.de/ new pages / TESLA/TTFnot2001.html

[17] G. R. Myneni. Physical and mechanical properties of single and large crystal high-RRR niobium. Proceedings of 12th Workshop on RF Superconductivity, SRF- 2005. 10-15 July 2005, Ithaca, USA.

[18] P.Kneisel. Performance of large grain and single crystal niobium cavities. Proceedings of 12th Workshop on RF Superconductivity, SRF- 2005. 10-15 July 2005, Ithaca, USA. 